\title{
Rotor Unbalance Detection in Electrical Induction Motors Using Orbital Analysis
}

\author{
José Juan Carbajal-Hernández , Luis P. Sánchez-Fernández, \\ Sergio Suárez-Guerra, and Ignacio Hernández-Bautista \\ Center of Computer Research - National Polytechnic Institute, Av. Juan de Dios Bátiz s/n, \\ Nueva Industrial Vallejo, Gustavo A. Madero, México D.F., C.P. 07738, México \\ \{jcarbajalh, 1sanchez, ssuarez\}@Cic.ipn.mx
}

\begin{abstract}
Deterioration in mechanical parts of a motor causes faults that generate vibrations. Those vibrations can be related with a different type of motor fault. In this work, we propose a new computational model for identifying rotor unbalance problems in electrical induction motors. Measured vibrations are preprocessed in order to create orbits which represent characteristic patterns. Those patterns are used in a recognition process using an artificial neural network. Experimental results using vibration signals extracted from real situations show a good performance and effectiveness of the proposed model, providing a new way for recognizing unbalance problems in induction motors.
\end{abstract}

Keywords: induction motor, fault, unbalance, rotor, orbit.

\section{Introduction}

Electrical induction motor is the most common engine used worldwide in industry. The normal usage of the engine generates deterioration in its physical parts over time, representing a problem when mechanical faults appear as a natural consequence. Induction motor fault detection is an important research field that uses different techniques for analyzing behaviors of the different conditions of the engine such as motor acoustic [1], electrical behaviors [2], orbital electromagnetic analysis [3] or motor vibrations [4]. In the literature, several technics for identifying some types of motor faults have been proposed using different mathematical theories such as spectral analyses, wavelet analysis, time warping, time frequency analysis, WignerVille distribution, etc. [5-9]. On the other hand, artificial intelligence has been proposed as an alternative way for recognizing some specific motor faults using technics such as artificial neural networks, Bayesian networks, support vector machine and fuzzy logic [10-15]. Thus, the importance of detecting and preventing motor faults before they appear is a challenge clearly recognized and some works are currently focused in this problem.

\footnotetext{
* Corresponding author.
} 
Motor vibrations are generated by the normal operation of the engine; however, when some faults appear, those vibrations increase significantly their levels and can generate some characteristic vibrations that can be studied. The most common induction motor fault presented is the rotor unbalance, and it is represented by an increment in vibration amplitudes. In this sense, we hypothesized that unbalance motor fault can be modeled using orbital analysis, where the amplitude of measured vibrations surely will modified a characteristic orbit; this behavior is used for recognizing the motor fault using a defined classifier. Although some works has been focused on using several learning models for motor fault recognizing, our main contribution is based on the pattern building, designing a new way for representing a motor fault. Hence, the core of this work is based on the feature extraction of vibration signals for building orbital patterns.

This work proposes a new computational model for identifying rotor unbalance faults in induction motors in three stages. First, some preprocessing steps are used in order to clean and prepare measured signals. Then, orbital patterns are generated indicating some features of a normal or a rotor unbalance operation. Finally, an artificial neural network is used for classifying those patterns with the mentioned problem.

\section{Rotor Unbalance Fault}

Failures in electrical induction motors can be classified in two types: mechanical or electrical fault. Those failures may occur frequently in the three main motor components: rotor, stator and bearings [10, 16-18]. In this work, we are mainly interested on studying the unbalance rotor fault due to it is most commonly presented fault. Therefore, rotor unbalance can be defined as the unequal mass distribution on the motor rotation center and in most of the cases; it is the main cause of vibration problems in induction motors (Fig. 1a). Unbalance occurs at a $1 \times \mathrm{rpm}$ frequency of the selected rotary member and the main harmonic presents amplitudes higher than normal [17]. Rotor unbalance can be classified as static or coupled unbalance (Fig. 1).

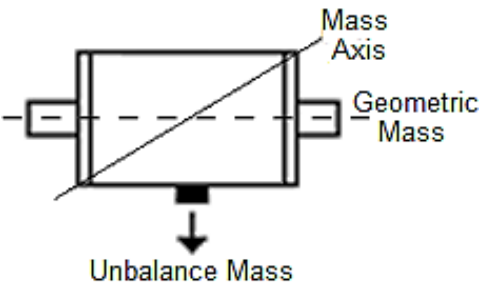

a)

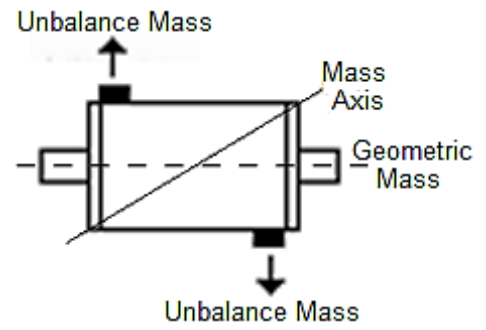

b)

Fig. 1. Rotor unbalance representations: a) static unbalance and b) couple unbalance 


\section{$3 \quad$ Signal Acquisition}

Mechanical motor faults can be detected if vibrations are monitored and measured continuously. A piezoelectric accelerometer sensor is used for measuring vibrations in the motor chassis. This device employs the piezoelectric effect of certain materials to measure dynamic changes in mechanical variables (e.g., acceleration, vibration, and mechanical shock). A piezoelectric accelerometer converts vibrations into an electrical signal which can be measured using an analog to digital converter [19]. As each motor have a different rotation speed, standards as ISO 10816 (1995) [20] and VDI 2056 (1964) [21] have established sampling frequency rates for motor measuring. According to them, the sampling frequency established was $50 \mathrm{kHz}$, being large enough to obtain a good quality signal over tested induction motors.

Orbital analysis are made using two signals plotted in a $(x, y)$ axis. Those signals are measured using two piezoelectric accelerometers placed orthogonally in the motor chassis. Fig. 2 shows the correctly accelerometers position.
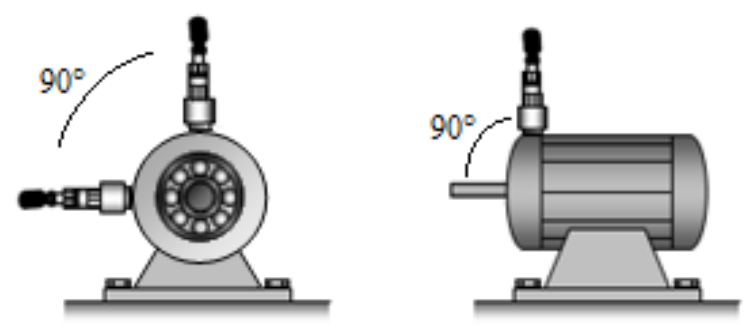

Fig. 2. Accelerometer placement at $90^{\circ}$ over the engine

Vibration signals obtained from induction motors contains several harmonics that can be considered as spurious information. In order to avoid those harmonics a previous signal treatment is needed in order to obtain good quality orbits. The following section explains this important process.

\section{$4 \quad$ Orbital Pattern Building}

In the literature, some works have focused their efforts on studying the induction motor behaviors using the orbital analysis across mechanical vibrations and electromagnetic forces [22, 3]. Generally, motor unbalance analyses has been made using spectral technics such as Fourier analysis. In this section, we propose an alternative way for transforming vibration input signals into characteristic patterns without using any type of spectral analysis (frequency domain). One advantage of the proposed model is that all processing steps are made in time domain, since no frequency transformations are needed. According to this, orbital analyses are used to create shapes using the time stamp of measured signals that remarks particular behaviors of an unbalance rotor problem. 


\section{Preprocessing}

In order to create an orbital pattern, some preprocessing steps are required in order to have a good quality orbit. First, each signal is preprocessed separately in order to preserve their particular features. An accelerometer measures signals in acceleration units. Fault orbits are plotted using different signal factors such as vibration amplitudes and distance between phases; hence, it is important to convert the current signal domain (acceleration) into a displacement domain; this unit conversion is computed by integrating the acceleration signal in time domain using the following definition [19]:

$$
\begin{aligned}
& v(t)=\int_{0}^{t} a(t) d t+v_{0} \\
& d(t)=\int_{0}^{t} v(t) d t+d_{0}
\end{aligned}
$$

where $a(t), v(t)$ and $d(t)$ are the acceleration, velocity and displacement signals; $v_{o}$ and $d_{o}$ are the initial velocity and displacement values respectively.

An induction motor generates several types of vibrations, but we are interested only in those produced by rotor unbalances. Undesirable vibrations considerably distort the shape of an orbit and they must be removed (Fig. 3). In this sense, signals must be filtered using a Butterworth passband filter. There is not a specific rule for using a digital filter; however, the Butterworth filter algorithm simplifies the implementation of this process. Therefore, signal filtering can be computed according to the following magnitude response [23, 24]:

$$
|H(\omega)|^{2}=\frac{1}{1+\left(\frac{c-\cos \omega}{\Omega_{0} \sin \omega}\right)^{2 \mathrm{~N}}}
$$

where $\omega=2 \pi f / f_{s}, f_{s}$ is the sampling frequency, $\Omega_{0}=\tan \left(\omega_{0} / 2\right)$ and $c$ can be expressed as follows:

$$
c=\frac{\sin \left(\omega_{p a}+\omega_{p b}\right)}{\sin \omega_{p a}+\sin \omega_{p b}}
$$

where $\omega_{p a}=2 \pi f_{p a} / f_{s}, \omega_{p b}=2 \pi f_{p b} / f_{s}$ and $\left[f_{p a}, f_{p b}\right]$ is the passband.

In this work, we used a bandpass frequency of $10 \mathrm{~Hz}$, this value provided a good quality in the orbit shape, removing undesirable harmonics.

Vibration signals generate continuously orbits with the same symmetry. However, for practical purposes, only one orbit is needed for detecting an unbalance in the rotor. In this sense, orbit isolation is made determining the Euclidian distance between the starting and ending points according with a low tolerance $(T)$ according with the following equation [23]:

$$
d=\sqrt{\left(x_{2}-x_{1}\right)^{2}+\left(y_{2}-y_{1}\right)^{2}}
$$


where $d$ is the distance between points, and $(x, y)$ are the orbit points coordinates respectively. There is not a specific rule for establishing the tolerance value. In this case, we used the median between point distances as follows:

$$
T=\frac{1}{n} \sum_{i=1}^{n} d_{i}
$$

Where $\mathrm{n}$ is the number of distances used. Finally, the extracted orbit is normalized into a $[0,1]$ range using the following expression:

$$
s_{1,2}(n)=\frac{d_{1,2}(n)}{\max \left\{\left|d_{1}(n)\right|,\left|d_{2}(n)\right|\right\}}, \quad \forall n=0,1,2, \ldots, N-1
$$

where $N$ is the number of points in the signal.
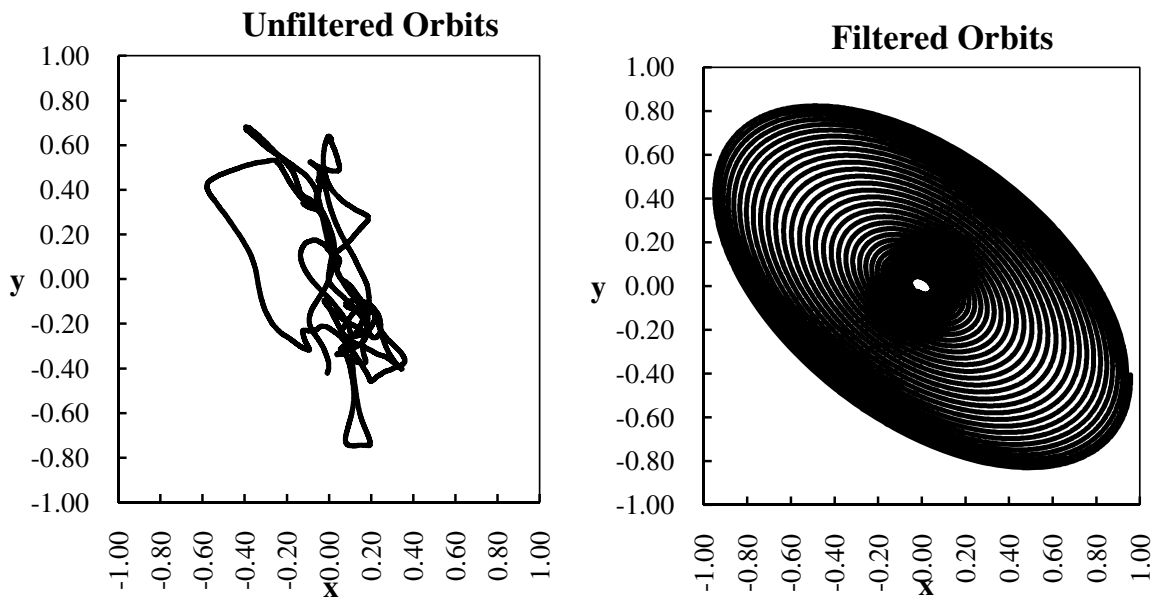

Fig. 3. Differences between filtered and unfiltered orbits, where the spurious harmonics has been removed

Finally, rotor unbalance problems can be directly seen in the shape of the orbit, where a perfect circular shape represents a good condition motor and elliptical shapes correspond to unbalanced rotor fault. Fig. 4 shows these concepts.

\section{Pattern Building}

According with the orbital signal analysis, orbit shapes where used for creating motor fault patterns. However, resulting signal orbits are not practical to be used in a neural network due to they have different lengths. In order to have uniform patterns, all orbits signals were resampled for having 100 points of length, where each one is a bidimensional pattern $(x, y)$. A training database with 324 patterns was created to be used in the learning phase of the ANN. Orbit shapes of this database were measured from different induction motors, which had 314 unbalance patterns and 10 patterns from good condition motors. 

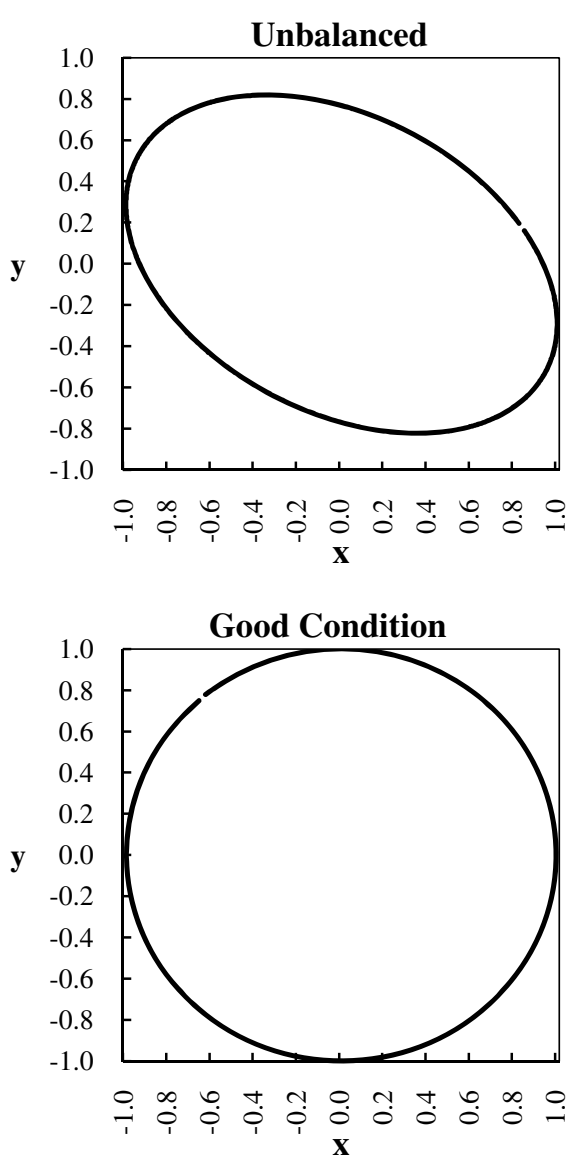

Fig. 4. Examples of orbit shapes that correspond to different motor faults

\section{$5 \quad$ Pattern Recognition and Experimental Results}

There are several technics for pattern recognition that can be used for orbits shape identification [1-11]. In this work, Artificial Neural Networks (ANN) are used as classifiers, because they have proved being a very effective learning model with high rates of effectiveness. The ANN architecture that better fitted to the problem context was of [200-150-2]. In this case, the inputs were fixed at 200 neurons, where the first half is for $x$-axis and the other half for $y$-axis. 1000 epochs where used for training the ANN having a MSE of $1 \times 10^{-10}$. The hidden layer was initialized between 2 and 200 neurons sequentially, where 150 neurons was the configuration with better results. It was used three sets to evaluate the performance of the ANN (dividing the training database for different purposes on the training process); one inside set for the training algorithm (484 partners from the available data to train), one validation set (97 patterns from the available data) and one final testing set to evaluate the final 
performance of the algorithm (64 partners from the available data), with correlation coefficients $(r)$ of $0.998,0.989$ and 0.995 respectively. The training process used by the ANN learning was the Levenberg-Marquardt algorithm.

Additionally, an experimental database was built and used for validating the performance of the proposed system as part of a recovering process. This database was built using different kind of motors and with different levels of unbalance. It is important to remark that this database was compounded by different motor measurements than those used in the database of the learning process. In this case, the size of the database was of 645 motor fault patterns as follows: 635 rotor unbalance patterns and 10 patterns of good condition motors.

The neural network was created using the training database and was tested with the experimental database with patterns of different unbalance and intensities. In the evaluation were extracted signals from real motors with history of failure. Table 1 shows the results of the recovering process using the proposed database. A second experiment was using the experimental database contaminated with Gaussian noise and a SNR of 10, having interesting results and where a low percentage of patterns were misclassified.

Table 1. Orbital pattern recognition results with the original experimental database and the Gaussian noise contaminated database

\begin{tabular}{|c|c|c|c|c|}
\hline \multirow[b]{2}{*}{ Experimental database } & \multirow[b]{2}{*}{$\begin{array}{c}\text { Number of } \\
\text { Patterns }\end{array}$} & \multicolumn{3}{|c|}{ ANN } \\
\hline & & $\begin{array}{l}\text { Recalled } \\
\text { patterns }\end{array}$ & Efficiency & Error \\
\hline Original patterns & 645 & 642 & $99.53 \%$ & $0.47 \%$ \\
\hline $\begin{array}{l}\text { Patterns contaminated } \\
\text { with Gaussian noise }\end{array}$ & 645 & 597 & $92.55 \%$ & $7.45 \%$ \\
\hline
\end{tabular}

\section{Discussion and Conclusions}

In this work, we propose a new computational model for detecting rotor unbalance problems in electrical induction motors. This model was implemented using the orbital analysis for creating pattern shapes that have characteristic features of the fault. Although several methodologies for detecting mechanical faults in induction motor have been developed, the proposed model represents a feasible and alternative way for motor misalignment fault detection. In this model, some preprocessing steps implemented before the ANN classification phase are needed for extracting the main features of signal patterns. One advantage of this work is that no spectral analyses were made, having low computational burden rates against conventional models. As an unbalance distorts considerably an orbit, a characteristic shape is built and it can be perfectly identified by a classifier such as an ANNs, reducing the complexity of the modeling step. It is important to remark that this paper provides a preliminary study of unbalance identification in induction motors using orbital pattern analysis, and future works are needed such as detection of more type of failures or model 
effectiveness increasing. Anyhow, this model can be used as an important tool for preliminaries motor analysis, when the good functioning of the machine is essential in critical time production.

\section{References}

1. Gaylard, A., Meyer, A., Landy, C.: Acoustic evaluation of faults in electrical machines. In: Electrical Machines and Drives, Conference Publication, vol. 412, pp. 147-150 (1995)

2. Yahya, L., Saleem, T., Hasan, B.: The application of Neural Network to Electrical Motor's Sound Recognition System. Journal of Engineering and Applied Sciences 7(2), 191-193 (2012)

3. Ha, K., Hong, J., Kim, G., Chang, K., Lee, J.: Orbital analysis of rotor due to electromagnetic force for switched reluctance motor. IEEE Transactions on Magnetics 36(4), 1407-1411 (2000)

4. Iorgulescu, M., Beloiu, R., Cazacu, D.: Vibration monitoring for electrical equipment faults detection using fast fourier transform. In: Proceedings of the 1 st International Conference on Manufacturing Engineering, Quality and Production Systems, vol. 1, pp. 34-38 (2009)

5. Kim, K., Parlos, A.: Induction motor fault diagnosis based on neuropredictors and wavelet signal processing. IEEE/ASME Transactions on Mechatronics 7(2), 201-219 (2002)

6. Climente, V., Antonino, J., Riera, M., Puche, R., Escobar, L.: Application of the WignerVille distribution for the detection of rotor asymmetries and eccentricity through highorder harmonics. Electric Power Systems Research 91, 28-36 (2012)

7. Liu, D., Zhao, Y., Yang, B., Sun, J.: A new motor fault detection method using multiple window S-method time-frequency analysis. In: International Conference on Systems and Informatics, pp. 2563-2566 (2012)

8. Yang, D.: Induction motor bearing fault diagnosis using Hilbert-based bispectral analysis. In: International Symposium on Computer, Consumer and Control, IS3C, Taichung, Taiwan (2012)

9. Zhen, D., Wang, T., Gu, F., Ball, A.: Fault diagnosis of motor drives using stator current signal analysis based on dynamic time warping. Mechanical Systems and Signal Processing 34(1-2), 191-202 (2013)

10. Chow, M.: Methodologies of using neural network and fuzzy logic technologies for motor incipient fault detection. WorldScientific, Singapore (1997)

11. Banerjee, T., Das, S.: Multi-sensor data fusion using support vector machine for motor fault detection. Information Sciences 217, 96-107 (2012)

12. Gardel, P., Morinigo, D., Duque, O., Pérez, M., Garcia.: Neural network broken bar detection using time domain and current spectrum data. In: Proceedings of the 20th International Conference on Electrical Machines, No. 6350234, pp. 2492-2497 (2012)

13. Liang, B., Iwnicki, S., Zhao, Y.: Application of power spectrum, cepstrum, higher order spectrum and neural network analyses for induction motor fault diagnosis. Mechanical Systems and Signal Processing 39(1-2), 342-360 (2013)

14. Sun, C., Duan, Z., Yang, Y., Wang, M., Hu, L.: The motor fault diagnosis based on neural network and the theory of D-S evidence. Advanced Materials Research 683, 881-884 (2013)

15. Zidani, F., Benbouzid, M., Diallo, D., Naït, M.: Induction Motor Stator Faults Diagnosis by a Current Concordia Pattern-Based Fuzzy Decision System. IEEE Transactions on Energy Conversion 18(4), 469-475 (2003) 
16. Palomino, E., Sánchez, A., Cabrera, J., Sexto, L.: Preliminary Diagnosis of Rotational Machinery. In: Experiences in the Implementation of a Predictive Maintenance Program and Certification of Human Resources in a Cuban Cement Industry. Advances in Vibration Control and Diagnostics, pp. 177-184. Polimetrica International Scientific Publisher (2006)

17. Arun, K., Mohanty, A.: Model based fault diagnosis of a rotor-bearing system for misalignment and unbalance under steady-state condition. Journal of Sound and Vibration 327(3-5), 604-622 (2009)

18. Chen, F., Jhe, S., Min, P., Wen, T.: Study of start-up vibration response for oil whirl, oil whip and dry whip. Mechanical Systems and Signal Processing, Elsevier 25(8), 31023115 (2011)

19. Han, S.: Retrieving the time history of displacement from measured acceleration signal. Journal of Mechanical Science and Technology 17(2), 197-206 (2003)

20. ISO 10816. Mechanical vibration: evaluation of machine vibration by measurements on non-rotating parts (1995)

21. VDI 2056. Standards of evaluation for mechanical vibrations of machines, Germany (1964)

22. Dongfeng, S., Lianfsheng, O., Ming, B.: Instantaneous purified orbit: a new tool for analysis of nonstationary vibration of rotor system. International Journal of Rotating Machinery 7(2), 105-115 (2001)

23. Proakis, J., Manolakis, D.: Tratamiento digital de señales, 4a edn. Pearson Education, vol. 1. España (2007)

24. Orfanadis, S.: Introduction to Signal Processing. Prentice Hall (2009) 